\title{
Magma migration and magmatic solitary waves in 3-D
}

\author{
Chris Wiggins ${ }^{1}$ and Marc Spiegelman
}

Lamont-Doherty Earth Observatory, Palisades NY

\begin{abstract}
Numerical studies of fluid flow in the mantle suggest that magma migration is an inherently time-dependent process that produces magmatic solitary waves from obstructions in melt flux. Previous work has considered one and two dimensional problems. Here we present the results of three dimensional calculations that utilize a new, efficient multigrid scheme. We demonstrate that one and two dimensional solitary waves are unstable and break up into sets of 3-D solitary waves which are perfectly spherical when propagating through a uniform porosity medium. While these waves are not solitons, their non-linear interactions are qualitatively similar. The solitary waves are highly opportunistic and establish efficient pathways for migration by linking up with nearby waves. When the initial condition is a random distribution of porosity, the porosity structure can organize into elongate, time-dependent channels formed from chains of solitary waves. These results are natural consequences of the assumptions that the matrix is permeable and viscously deformable. We suggest that solitary waves are likely to exist in the mantle and may contribute to the episodicity of mantle magmatism.
\end{abstract}

\section{Introduction}

In an attempt to understand the ascent of magma in the Earth's mantle, we have studied the equations governing the flow of a low viscosity fluid in a viscously deformable, permeable matrix [McKenzie, 1984; Scott and Stevenson, 1984; Scott and Stevenson, 1986]. Considerable numerical and analytic work has demonstrated a wide range of behaviour inherent in these equations, ranging from magmadriven mantle convection to non-linear waves [see Spiegelman, 1993c for review]. Soon after their derivation, the 1-D equations were shown to exhibit analytic solitary wave solutions that share several of the features of solitons, specifically amplitude-dependent velocity and non-linear interactions upon collision [Richter and McKenzie, 1984; Scott and Stevenson, 1984] although the 1-D waves were shown not to be solitons [Barcilon and Richter, 1986]. Later, the solution for a single solitary wave was generalized to 2 and 3 dimensions with the help of some numerics [Barcilon and Lovera, 1989; O. Lovera, pers. comm. 1993]. Numerical and analytical work by Scott and Stevenson [1986] and Barcilon and Lovera [1989] showed that the 1-D solution was unstable

${ }^{1}$ Now at Physics Department, Princeton University

Copyright 1995 by the American Geophysical Union.

Paper number 95GL00269

0094-8534/95/95GL-00269\$03.00 in 2 dimensions and broke up into cylindrical 2-D solitary waves. While it was suggested that these waves would be unstable in three dimensions, the full time-dependent behaviour of these equations in 3-D remained unknown, owing to the taxing computational task of simulation.

We have now calculated the 3-D time-dependent behaviour of the 1,2, and 3-D solutions using an efficient multigrid algorithm. Our results indicate that the lower dimensional solutions are unstable in 3-D and evolve into the 3-D solitary waves which are perfectly spherical in a homogeneous medium. More generally, the solitary waves form a means for transmitting variations in fluid flux through the mantle and we illustrate the behaviour and consequences of the nonlinear interactions between solitary waves.

\section{Model Equations and Numerics}

A detailed derivation of the equations of magma migration can be found in McKenzie [1984]. Spiegelman [1993a,b] however, rewrites the full equations in a more tractable form that separates matrix shear deformation from volumetric deformation (i.e. compaction and dilation of the matrix). To illustrate the simplest non-linear wave behaviour in 3-D, here we will neglect matrix shear, melting and consider the limit of small porosity. Under these conditions, the dimensionless equations reduce to

$$
\begin{gathered}
\frac{\partial \phi}{\partial t}=\mathcal{C} \\
-\nabla \cdot k_{\phi} \nabla \mathcal{C}+\mathcal{C}=-\nabla \cdot k_{\phi} \mathbf{k}
\end{gathered}
$$

where $\phi$ is the porosity, $C \equiv \nabla \cdot \mathrm{V}$ is the 'compaction rate' or volumetric strain rate of the matrix, $V$ is the matrix velocity, $\mathbf{k}$ is the unit vector in the vertical and $k_{\phi} \propto \phi^{n}$ is the permeability. Here we use $n=3$ because these waves have analytic expressions in 1-D [see Barcilon and Richter, 1986; Barcilon and Lovera, 1989]. Equation (1) states that changes in porosity are due to changes in matrix volume. Equation (2) states that volume changes of the matrix are driven by variations in the gravity driven flux (RHS) but are resisted by viscous stresses in the matrix (1 st term LHS). The derivation and importance of the viscous stress term for modifying fluid flow is discussed in detail in Spiegelman [1993a,b]. This term becomes important when changes in fluid flux occur on a length scale comparable to the 'compaction length' $(\sim 100-$ $1000 \mathrm{~m}$ ) and controls the development of solitary waves and the flow of melt around obstacles. While the compaction length may be small, Spiegelman [1993a,b] shows that the viscous term can affect fluid flow on scales much larger than the compaction length.

These equations are readily solved numerically. Equation (1) is solved with a flux-conservative leap-frog scheme. Equation (2) is solved using an iterative multigrid scheme 

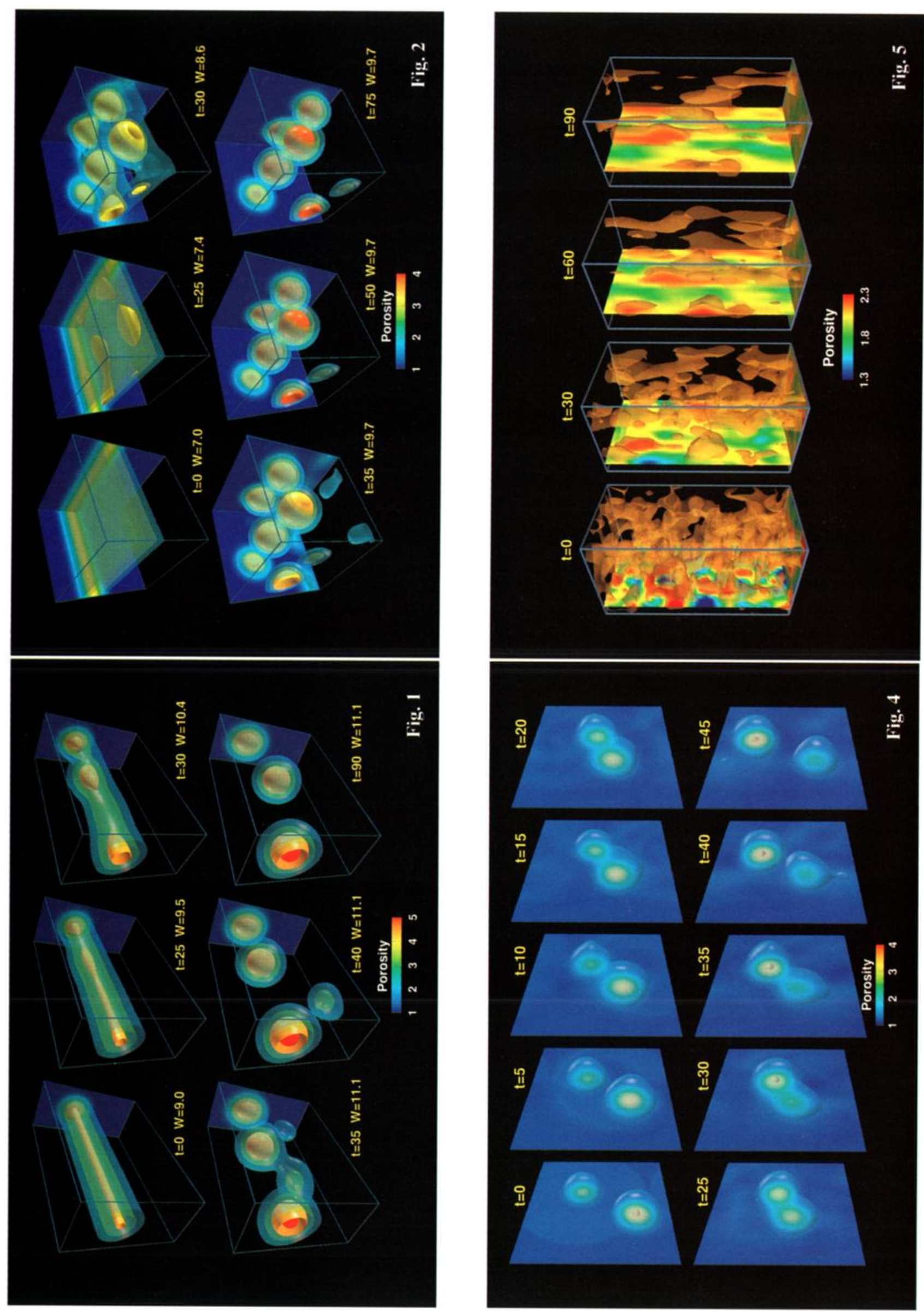
Figure 1. Evolution of a cylindrical 2-D solitary wave in 3-D. The initial condition is an analytic 2-D solitary wave of dimensionless speed $c=9$ (Amplitude $\approx 4$ ) which has been perturbed by Gaussian white noise of magnitude $10^{-3}$ times the background porosity. In 3-D the cylindrical waves are unstable and break up into larger spherically symmetric waves. When the waves are fully formed, they propagate without further change of shape. Each frame shows the position of surfaces of constant porosity which are.scaled relative to the background porosity ( $\phi_{\max } \approx 5 \times$ background). All waves propagate upwards, however, this calculation (and Figure 2) are performed in a frame that tracks the fastest moving wave. $W$ is the dimensionless speed of the frame scaled to the velocity of melt in the background porosity. The boxes are periodic in both horizontal directions and have constant porosity upper boundaries. Box is $96 \times 32 \times 64$ compaction lengths (defined for the background porosity), $(97 \times 33 \times 65$ grid points).

Figure 2. Evolution of a single 1-D solitary wave in 3-D which goes unstable directly to spherical waves. The 2-D waves are not only unstable; they are not even a transitional waveform in three dimensions. Boundary conditions and scalings are the same as in Figure 1. Box is $64 \times 64 \times 64$ compaction lengths ( $65^{3}$ grid points).

Figure 4. Interaction of two 3-D waves on oblique collision. Each frame shows both a constant-porosity surface and a contoured vertical cross section through the mid-plane of the two waves. This calculation is done in a frame moving at the mean velocity of the two waves and shows the classic soliton-like phase-shift on collision. More physically, the individual solitary waves retain their shape until they are within a few compaction lengths of each other and then fluid from the lower wave seeks the higher permeability pathway afforded by the upper wave. The upper wave inflates at the expense of the lower wave and they appear to trade places. The 3-D waves however are not solitons and the larger wave tends to grow during collisions. Boundaries are periodic in all directions. Box is $64^{3}$ compaction lengths.

Figure 5. Evolution of an initially random distribution of porosity in a periodic box. This calculation is done in a frame fixed to the matrix $(W=0)$. Porosity waves rapidly circulate through the box, re-organizing into elongate channel structures of more 'spindle' shaped solitary waves. The channels are long lived but remain strongly episodic throughout the run. Box is $32 \times 32 \times 64$ compaction lengths.

[e.g. Press et al., 1992]. The resulting algorithm is secondorder accurate in space and time and extremely efficient because it retains the convergence behaviour of multigrid schemes where the number of numerical operations scale linearly with the number of grid points. We have tested our numerical schemes against the solutions of Barcilon and Lovera [1989] who calculate the shape of single solitary waves in 1,2 and 3-D. These waveforms should propagate at a constant velocity without changing shape. Our numerical algorithm preserves the theoretical amplitude and phase velocity to about $0.1 \%$ : We next reproduced the work of Scott and Stevenson [1986], verifying the instability of the 1-D solution in 2 dimensions and the development of cylindrically symmetric 2-D solitary waves.

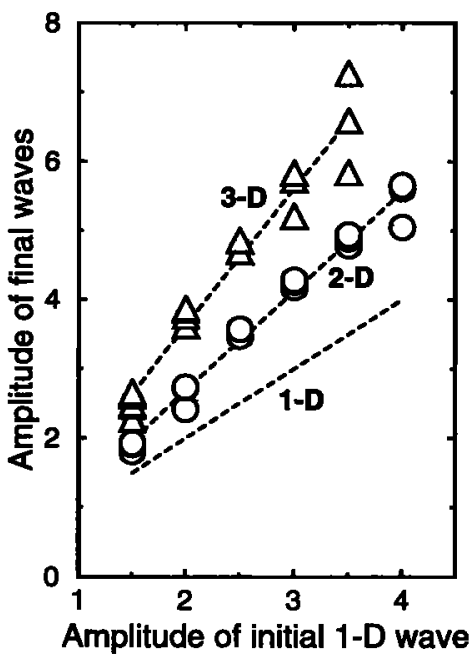

Figure 3. Summary of results of a series of runs to quantify the evolution of 1-D solitary waves in 2-D (circles) and 3-D (triangles). The symbols show the maximum porosity of the final $n-D$ waves that are produced in each run as a function of their initial 1-D amplitude. Note each run produces several large solitary waves of slightly varying amplitude.

\section{Results}

Extending the analysis to 3 dimensions, we investigated the behaviour of one and two dimensional waves in 3-D. Both 1-D and 2-D waves are unstable and rapidly break up into spherically symmetric 3-D solitary waves (Figures 1 and 2). In particular, the 1-D waves go directly to spheres and the 2-D waves are not even transitional waveforms. Figure 1 illustrates the general behaviour of the instability. After an initial period of propagation, local excesses of porosity begin to form resulting in a 'doming' of the porosity isosurfaces. Calculation of the fluid flux near these excesses shows that viscous stresses drive melt preferentially toward the peak of the domes causing the waves to grow. The solitary waves stop growing, however, when they can no longer be supplied by melt, which occurs when the waves are separated by more than a few compaction lengths. At this point the individual waves propagate without change of shape. In general, a single 1-D wave will break up into a family of higher dimensional waves with similar, but not identical, amplitudes. Preliminary linear analysis suggests that the final plan-form of the waves and the spread in amplitudes is sensitive to the size of the box and the structure of the noise. Nevertheless, the mean amplitude of solitary waves appears to be well determined. Figure 3 summarizes the results of a series of runs in 2-D and 3-D showing the final amplitudes of the largest waves at the end of each run as a function of the amplitude of the initial 1-D wave. For a given 1-D wave, the 3-D waves are always larger and faster moving than the cylindrical wave that develops in 2-D. While we are developing a scaling argument to quantify this behaviour, linear regression shows that $A_{f} \approx 2^{(D-1) / 2} A_{i}$ is a useful relation between the mean amplitude of the final waves, $A_{f}$, and the initial amplitude $A_{i} . D$ is the maximum physical dimension.

The development of 3-D solitary waves from arbitrary excesses of porosity is robust. As long as the solitary waves remain separated by a few compaction lengths in a uniform medium, they propagate as perfectly spherical waves. While this result might suggest that spherical solitary waves would be the preferred waveform in the mantle, it is important to 
note that the waves are quite opportunistic and will locally change their shape to exploit variations in permeability that allow for more efficient melt transport. Figure 4 shows the interaction of two 3-D waves on collision. Until the waves are within a few compaction lengths of each other, they propagate independently. However, when they are close enough that the high pressure region at the leading edge of the lower wave "sees" the higher permeability region in the upper wave, fluid flows preferentially from the lower wave to the upper one until they appear to trade places.

A similar interaction is also seen in solitons, which are infinitely-conservative solitary waves. We stress, however, that these magma waves are not solitons, as they have only two, rather than an infinite number of, conserved quantities [Barcilon and Richter, 1986]. To verify that the solutions do not result from an infinitely integrable system, we allowed two 3-D solitary waves to interact repeatedly in a periodic environment. Unlike soliton collisions, the larger wave grows at the expense of the smaller wave and the two waves tend to become aligned vertically. After several collisions, the smaller wave is split in two. This behaviour is typical. Multi-dimensional particle-like solitary waves are not known to scatter elastically, but generally radiate upon collision (D. McLaughlin, pers. comm. 1993)

Because of this non-conservative behaviour, the placetrading interaction provides a means for organizing melt flow. Figure 5 shows the evolution of an initially random porosity distribution in a periodic box. Because gravity is the only driving force, this initial condition organizes into columnar channels of solitary waves through a large number of nonlinear interactions. In this example, the channels are long lived but remain strongly episodic with elongate "spindle" shape solitary waves that propagate along the higher permeability region defined by the channel. While some of the organization stems from the use of periodic boundary conditions, the general coalescence and alignment of the melt flow paths seems characteristic of wave-wave interactions.

\section{Discussion}

These results support the inferences of 1 and 2-D problems: the equations of magma migration are highly timedependent, and variations in melt flux propagate as non-linear solitary waves. In uniform media, the preferred waveform in 3-D is a spherically symmetric wave. In heterogeneous media, however, the waves may locally change their shape to exploit variations in permeability, although they tend to remain as compact blobs. This ability of the solitary waves to adjust to local circumstances appears to be characteristic and persists even when additional processes are included. While we have only considered the most basic 3-D problems here, other work suggests that solitary waves are robust even in the presence of matrix shear and melting. Scott [1988] showed that 2-D solitary waves are unaffected by the additional diapiric shear flow of the matrix induced by the waves.
Spiegelman [1993a,b,c] quantified the affects of melting and freezing on 1-D waves and showed that the waves change amplitude in response to changes in the melt flux but are not dissipated. More recent 2-D solutions of the full equations in a mid-ocean ridge geometry show that spatial variations in melting rate can excite solitary waves even when there is significant shear and melting. Finally, preliminary calculations using a porosity-dependent solid viscosity also produce stable solitary waves. More work needs to be done to extend these results to 3-D and to investigate additional processes. However, all of the work to date suggests that, while the shape of the waves may change, their existence depends only on the conditions that the matrix is permeable and viscously deformable. As these conditions are reasonable for partially molten regions of the upper mantle, we suggest that porosity waves are likely to exist there in some form, and may contribute to the episodicity of mantle magmatism.

Acknowledgments. C.W. thanks Mike Shelley and Dave Muraki for their insight and interest. We also thank Oscar Lovera for advice and mailing his personal notes. This is LDEO contribution 5312.

\section{References}

Barcilon, V. and O. Lovera, Solitary waves in magma dynamics, $J$. Fluid Mech., 204, 121-133, 1989.

Barcilon V. and F. M. Richter, Non-linear waves in compacting media, J. Fluid Mech., 164, 429-448,1986.

McKenzie, D., The generation and compaction of partially molten rock, J. Petrol., 25, 713-765, 1984.

Press. W.H., S.A. Teukolsky, W.T. Vetterling, and B.P. Flannery, Numerical Recipes in Fortran, 2nd ed., Cambridge University Press, Cambridge, U.K., 1992.

Richter, F. and D. McKenzie, Dynamical Models for melt segregation from a deformable matrix, J. Geol., 92, 729-740, 1984.

Scott, D. and D. Stevenson, Magma solitons, Geophys. Res. Lett., $11,1161-1164,1984$.

Scott, D. and D. Stevenson, Magma ascent by porous flow, J. Geophys. Res., 91, 9283-9296, 1986.

Scott, D., The competition between percolation and circulation in a deformable porous medium, J. Geophys. Res., 93, 6451-6462, 1988.

Spiegelman, M., Flow in deformable porous media. Part 1. Simple analysis, J. Fluid Mech., 247, 17-38, 1993a.

Spiegelman, M., Flow in deformable porous media. Part 2. Numeri$\mathrm{cal}$ analysis - the relationship between shock waves and solitary waves, J. Fluid Mech., 247, 39-63, 1993 b.

Spiegelman, M., Physics of melt extraction: Theory, implications and applications, Trans. Roy. Soc. London, 342, 23-41, $1993 \mathrm{c}$.

M. Spiegelman, Lamont-Doherty Earth Observatory, Palisades, NY 10964 (e-mail: mspieg@ldeo.columbia.edu)

C. Wiggins, Physics Dept., Princeton University, Princeton NJ 08544 (e-mail: cwiggins@phoenix.princeton.edu)

(received September 19, 1994; accepted November 29, 1994.) 